\title{
Erratum: 'Theory of two-section laser amplifiers'
}

Optical and Quantum Electronics 21, S15-S31 (1989)

Equations (32), (33), (A11) and (A12) in the above paper contain errors. The correct versions are given below:

$$
\begin{gathered}
\left(I_{\mathrm{lav}}\right)_{\mathrm{spon}}=\beta E\left[\frac{\left(\mathrm{e}^{g L_{1}}-1\right)\left[\chi\left(1-\mathrm{e}^{-\alpha L_{2}}\right) R_{\mathrm{sp} 2} / \alpha+\xi_{1} R_{\mathrm{sp1}} / g\right]}{2\left(1-R_{1} R_{2} \mathrm{e}^{2 g L_{1}-2 \alpha L_{2}}\right)}-R_{\mathrm{sp} 1} / g\right] \\
\left(I_{2 \mathrm{av}}\right)_{\mathrm{spon}}=(\beta E / \alpha)\left[\frac{\left(1-\mathrm{e}^{-\alpha L_{2}}\right)\left[\chi\left(\mathrm{e}^{g L_{1}}-1\right)\left(L_{1} / L_{2}\right) R_{\mathrm{spl}}-\xi_{2} R_{\mathrm{sp} 2}\right]}{2\left(1-R_{1} R_{2} \mathrm{e}^{2 g L_{1}-2 \alpha L_{2}}\right)}+R_{\mathrm{sp} 2}\right] \\
\frac{I_{\mathrm{I}}^{-}\left(-L_{1}\right)}{\beta E}=\frac{\mathrm{e}^{g L_{1}}\left(1-\mathrm{e}^{-\alpha L_{2}}\right)\left(1+R_{2} \mathrm{e}^{-\alpha L_{2}}\right) R_{\mathrm{sp} 2} / \alpha+\left(\mathrm{e}^{g L_{1}}-1\right)\left(1+R_{2} \mathrm{e}^{g L_{1}-2 \alpha L_{2}}\right) R_{\mathrm{sp} 1} / g}{2\left(1-R_{1} R_{2} \mathrm{e}^{2 g L_{1}-2 \alpha L_{2}}\right)} \\
\frac{I_{2}^{+}\left(L_{2}\right)}{\beta E}=\frac{\left(1-\mathrm{e}^{-\alpha L_{2}}\right)\left(1+R_{1} \mathrm{e}^{2 g L_{1}-\alpha L_{2}}\right) R_{\mathrm{sp} 2} / \alpha+\mathrm{e}^{-\alpha L_{2}}\left(\mathrm{e}^{g L_{1}}-1\right)\left(1+R_{1} \mathrm{e}^{g L_{1}}\right) R_{\mathrm{sp} 1} / g}{2\left(1-R_{1} R_{2} \mathrm{e}^{2 g L_{1}-2 \alpha L_{2}}\right)}
\end{gathered}
$$

The results given in the paper are in no way affected by these corrections. I am indebted to $\mathrm{P}$ E Barnsley for renewing my interest in the subject of this paper.

M. J. Adams

BT Laboratories

Martlesham Heath

Ipswich IP5 7RE 